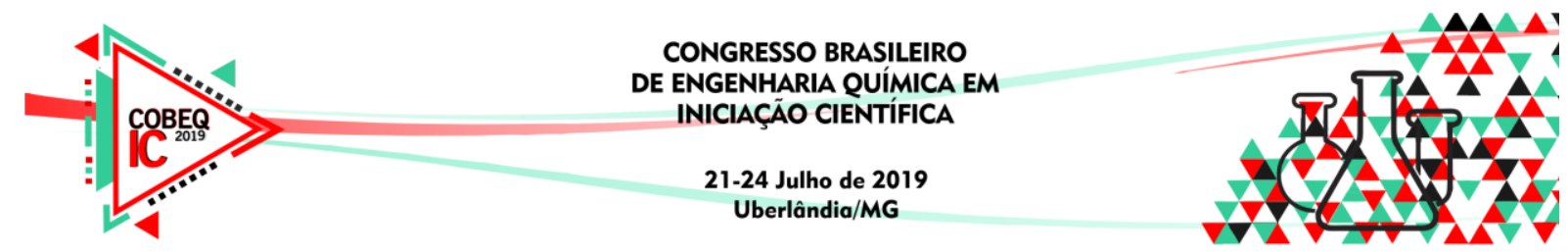

\title{
BIOSSORÇÃO DO CORANTE AZUL DE METILENO A PARTIR DE BIOMASSA INATIVA DE Aspergillus oryzae
}

\author{
L. R. V. LITORDI ${ }^{1}$, G. E. SANTOS ${ }^{1}$, F. H. M. SOUZA ${ }^{1}$ e G. S. S. ANDRADE ${ }^{1}$ \\ ${ }^{1}$ Universidade Federal de Alfenas, Instituto de Ciência e Tecnologia \\ E-mail para contato: grazielle.andrade@unifal-mg.edu.br
}

\begin{abstract}
RESUMO - O presente trabalho estuda a eficiência da biomassa inativa do fungo filamentoso Aspergillus oryzae na biossorção do corante Azul de Metileno em solução aquosa. Para isso, foram realizados ensaios de adsorção em batelada a $30^{\circ} \mathrm{C}, 170 \mathrm{rpm}$ utilizando $2 \mathrm{~g} \mathrm{~L}^{-1}$ de biossorvente em diferentes concentrações do corante. A influência do tempo de contato entre adsorvente e adsorbato foi avaliada por meio de retiradas de amostras periódicas para avaliação da capacidade de adsorção. Os modelos de Pseudo-primeira ordem, Pseudo-segunda ordem e Elovich foram ajustados aos dados experimentais para avaliar a cinética de adsorção. O comportamento do equilíbrio foi estudado mediante o ajuste das isotermas de Freundlich e Langmuir. Os resultados evidenciaram que a reação de adsorção segue o modelo cinético de Pseudo-segunda ordem e a isoterma de equilíbrio que melhor se ajustou aos dados foi a de Freundlich, indicando um sistema de adsorção não ideal, que ocorre em multicamadas sob uma superfície heterogênea.
\end{abstract}

\section{INTRODUÇÃO}

Indústrias têxteis, alimentícia e de papel utilizam grande quantidade de água e corantes em seus processos, os quais podem apresentar moléculas complexas e de difícil degradação. Dentre os diversos corantes utilizados industrialmente, destaca-se o azul de metileno, um corante catiônico, empregado no tingimento de algodão, lãs, papel e tinturas para cabelos. Devido ao seu grande uso e a sua periculosidade, faz-se necessário tratar o efluente gerado antes de ser descartado em corpos hídricos (Oliveira et al., 2013).

Diversos métodos de tratamento físico-químicos são empregados no tratamento de águas residuais contendo corantes, sendo a adsorção considerada uma das técnicas mais eficazes (Anastopoulos, Kyzas, 2015). Nesse aspecto, com objetivo de produzir um adsorvente de baixo custo de origem biológica o presente trabalho estudou o potencial da biomassa fúngica inativa de Aspergillus oryzae no processo de biossorção do corante Azul de Metileno.

\section{MATERIAIS E MÉTODOS}

\subsection{Preparação Do Biossorvente e Solução Corante}




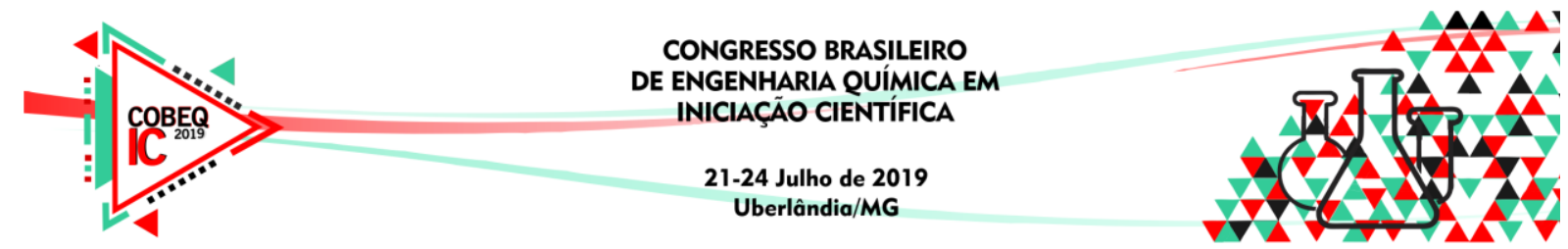

O fungo Arpergillus oryzae URM 5618 (Micoteca UFPE) foi empregado como biossorvente e preparado conforme Majundar et al. (2010) modificado. Uma suspensão de 0,2 $\mathrm{mL}$ de esporos do fungo foi inoculado em $100 \mathrm{~mL}$ de meio de cultura líquido composto por caldo batata:dextrose $\left(10: 1 \mathrm{~m} \mathrm{v}^{-1}\right)$ previamente autoclavados $\left(121^{\circ} \mathrm{C} / 20 \mathrm{~min}\right)$. Os Erlenmeyers de $250 \mathrm{~mL}$ contendo meio e inóculo foram incubados em shaker $(170 \mathrm{rpm})$ por $72 \mathrm{~h}$ a $30^{\circ} \mathrm{C}$. Após esse período, os Erlenmeyers foram autoclavados $\left(121^{\circ} \mathrm{C} / 20 \mathrm{~min}\right.$.), sendo que a biomassa inativa foi separada do meio de cultura por filtração a vácuo, lavada com água destilada e seca em estufa $\left(60^{\circ} \mathrm{C} / 24\right.$ horas $)$. As soluções de corante foram preparadas pela diluição do Azul de Metileno (Synth) em água destilada em diferentes concentrações (50, 100,200 e $300 \mathrm{mg} \mathrm{L}^{-1}$ ).

\subsection{Ensaios De Biossorção}

Os ensaios de biossorção foram realizados em Erlenmeyers de $250 \mathrm{ml}$ contendo $50 \mathrm{ml}$ de solução de Azul de Metileno e $2 \mathrm{~g} \mathrm{~L}^{-1}$ de biomassa a $30^{\circ} \mathrm{C}, 170 \mathrm{rpm}$ e por um período máximo de 48h. Para cada concentração de solução de corante estudada, amostras periódicas foram coletadas, centrifugadas (3500 rpm/10 min) e submetidas a leitura em espectofotômetro UV-Vis (Edutec - modelo EEQ901A-B) a $552 \mathrm{~nm}$. Ensaios controle (sem biomassa) foram realizados nas mesmas condições operacionais. Para a análise da capacidade adsortiva do material foi calculada a quantidade adsorvida (q) apresentada na Equação 1 (Majundar et al., 2010)

$q=\frac{C_{i}-C_{f}}{m} V$

Em que: $q$ é a quantidade adsorvida $\left(\mathrm{mg} \mathrm{g}^{-1}\right) ; C_{i}$ é a concentração inicial do corante $\left(\mathrm{mg} \mathrm{L}^{-1}\right)$; $C_{f}$ é a concentração no equilíbrio $\left(\mathrm{mg} \mathrm{L}^{-1}\right) ; m$ é massa de biossorvente $(\mathrm{g})$ e $V$ é o volume da solução de corante (L).

\subsection{Estudo Cinético e Isotermas De Adsorção}

Para avaliação da cinética de biossorção foram utilizados os modelos de Pseudoprimeira ordem, Pseudo-segunda ordem, Elovich (Saba et al., 2016), conforme equações 2, 3 e 4 , respectivamente.

$$
\begin{aligned}
& q(t)=q_{e q}\left(1-e^{-k_{1} t}\right) \\
& q(t)=\frac{q_{e q}^{2} k_{2} t}{1+q_{e q} k_{2} t} \\
& q(t)=\frac{1}{\beta} \ln (\alpha \beta)+\frac{1}{\beta} \ln (t)
\end{aligned}
$$

Em que $q_{e q}$ é a quantidade adsorvida no equilíbrio $\left(m g g^{-1}\right), k_{1}$ a constante de velocidade da reação $\left(\mathrm{min}^{-1}\right), k_{2}$ a constante de velocidade de reação de Pseudo-segunda ordem $\left(g \mathrm{mg}^{-1} \mathrm{~min}^{-1}\right), \alpha$ é uma constante relacionada à taxa de quimiossorção e $\beta$ é uma constante que representa a extensão da cobertura superficial 


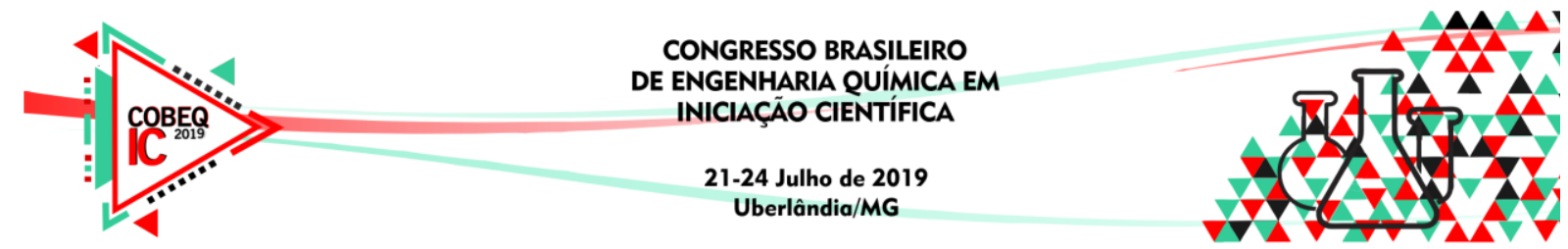

Para avaliação do equilíbrio de biossorção foram empregados os modelos de isoterma de Langmuir e Freundlich (Saba et al., 2016), conforme equações 5 e 6, respectivamente.

$q_{e q}=\frac{q_{m a ́ x} b C_{e q}}{1+b C_{e q}}$

$q_{e q}=K_{F} C_{e q}^{1 / n} q(t)=q_{e q}\left(1-e^{-k_{1} t}\right)$

Em que: $b$ é a constante de equilíbrio de Langmuir $\left(L m g^{-1}\right) q_{m a ́ x}$ a quantidade máxima de corante adsorvido por unidade de massa de adsorvente, $\left(m g g^{-1}\right), C_{e q}$, a concentração de equilíbrio do corante $\left(m g l^{-1}\right), K_{F}$ é a constante de Freundlich que representa a capacidade de adsorção multicamada $\left(m g g^{-1}\right)$ e $n\left(m g L^{-1}\right)$ representa a intensidade de adsorção.

\section{RESULTADOS E DISCUSSÕES}

\subsection{Estudo Cinético}

A quantidade de corante adsorvido pelo biossorvente em soluções de diferentes concentrações está representada pela Figura 1. Observa-se para todas as concentrações que a biossorção foi crescente nas primeiras 24 horas de análise, devido a maior disponibilidade de sítios ativos livres no biossorvente. A maior capacidade de biossorção (38 $\left.\mathrm{mg} \mathrm{g}^{-1}\right)$ foi obtida com a solução de $300 \mathrm{mg} \mathrm{L}^{-1}$ de azul de metileno.

Figura 1 - Quantidade de corante adsorvido pelo biossorvente, $q_{t}$, em função do tempo para diferentes concentrações de azul de metileno.

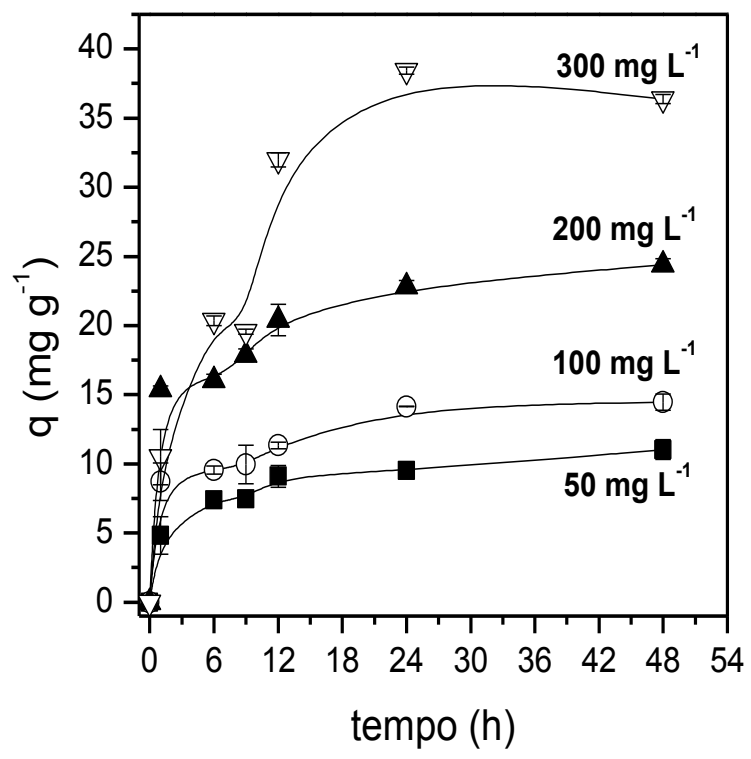

A Tabela 1 apresenta os parâmetros dos modelos cinéticos ajustados aos dados experimentais. Observa-se que para todas as concentrações de corante, o modelo de Pseudosegunda ordem $\left(\mathrm{R}^{2}>0,99\right)$ foi o que melhor se ajustou aos dados experimentais. 


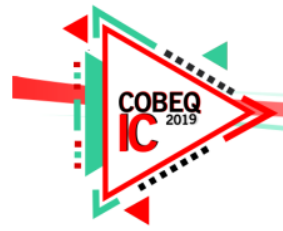

Tabela 1 - Parâmetros cinéticos ajustados aos dados experimentais em diferentes concentrações de Azul de Metileno

\begin{tabular}{|c|c|c|c|c|c|}
\hline \multirow{2}{*}{ Modelos Cinéticos } & \multirow{2}{*}{ Parâmetros cinéticos } & \multicolumn{4}{|c|}{ Concentração inicial ( $\left.\mathrm{mg} \mathrm{g}^{-1}\right)$} \\
\hline & & $\overline{c 50}$ & 100 & 200 & 300 \\
\hline \multirow{3}{*}{ Pseudo-primeira ordem } & $\mathrm{k} 1\left(\min ^{-1}\right)$ & 0,0320 & 0,0348 & 0,0454 & 0,0732 \\
\hline & qe $\left(\mathrm{mg} \mathrm{g}^{-1}\right)$ & 2,4831 & 3,9820 & 6,0353 & 18,4120 \\
\hline & $\mathrm{R}^{2}$ & 0,3266 & 0,7495 & 0,7230 & 0,7255 \\
\hline \multirow{3}{*}{ Pseudo-segunda ordem } & $\mathrm{k}_{2}\left(\mathrm{~g} \mathrm{mg}^{-1} \mathrm{~s}^{-1}\right)$ & 0,0184 & 0,0171 & 0,0118 & 0,0031 \\
\hline & $\mathrm{q}_{\mathrm{e}}\left(\mathrm{mg} \mathrm{g}^{-1}\right)$ & 11,9332 & 15,6495 & 25,9740 & 43,6681 \\
\hline & $\mathrm{R}^{2}$ & 0,9964 & 0,9958 & 0,9983 & 0,9665 \\
\hline \multirow{3}{*}{ Elovich } & $\mathrm{a}\left(\mathrm{mg} \mathrm{g}^{-1} \min ^{-1}\right)$ & 9,0734 & 24,7572 & 68,2616 & 11,4142 \\
\hline & $\mathrm{b}\left(\mathrm{g} \mathrm{mg}^{-1}\right)$ & 0,4739 & 0,4207 & 0,2757 & 0,0968 \\
\hline & $\mathrm{R}^{2}$ & 0,9387 & 0,9255 & 0,9495 & 0,8497 \\
\hline
\end{tabular}

\subsection{Isotermas De Adsorção}

A partir dos dados de equilíbrio obtidos experimentalmente, analisou-se o processo de biossorção através do estudo das isotermas expressas na Figura 2.

Figura 2 - Isotermas de adsorção.

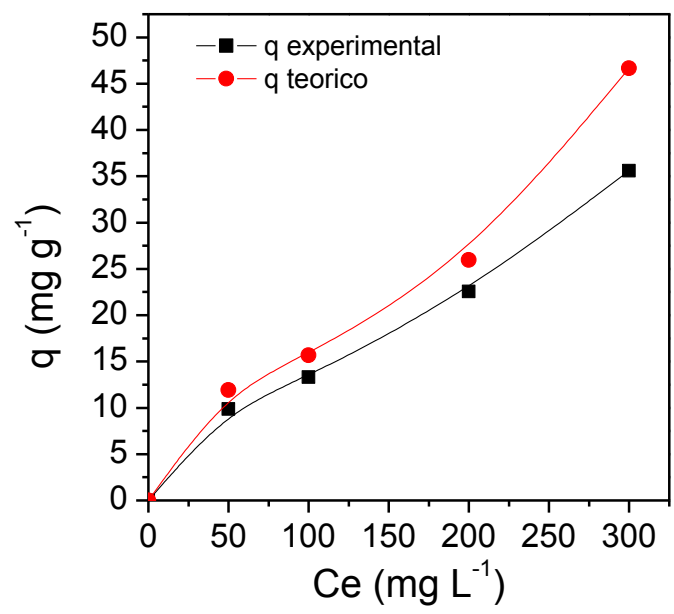

Para análise das interações entre adsorvato e adsorvente no processo de biossorção ajustou-se os dados experimentais de equilíbrio aos modelos linearizados das isotermas de Langmuir e Freundlich.

Tabela 2 - Parâmetros de equilíbrio para as isotermas de Langmuir e Freundlich

\begin{tabular}{|c|c|c|}
\hline Modelo de Isoterma & Parâmetros de equilíbrio & Valores \\
\hline \multirow{3}{*}{ Langmuir } & $\mathrm{q}_{\max }\left(\mathrm{mg} \mathrm{g}^{-1}\right)$ & 53,76 \\
\cline { 2 - 3 } & $\mathrm{K}_{\mathrm{L}}\left(\mathrm{L} \mathrm{mg}^{-1}\right)$ & 0,01 \\
\cline { 2 - 3 } & $\mathrm{R}^{2}$ & 0,7793 \\
\hline \multirow{3}{*}{ Freundlich } & $\mathrm{K}_{\mathrm{F}}\left(\mathrm{mg} \mathrm{g}^{-1}\right)$ & 1,36 \\
\cline { 2 - 3 } & $\mathrm{N}$ & 1,67 \\
\cline { 2 - 3 } & $\mathrm{R}^{2}$ & 0,9609 \\
\hline
\end{tabular}




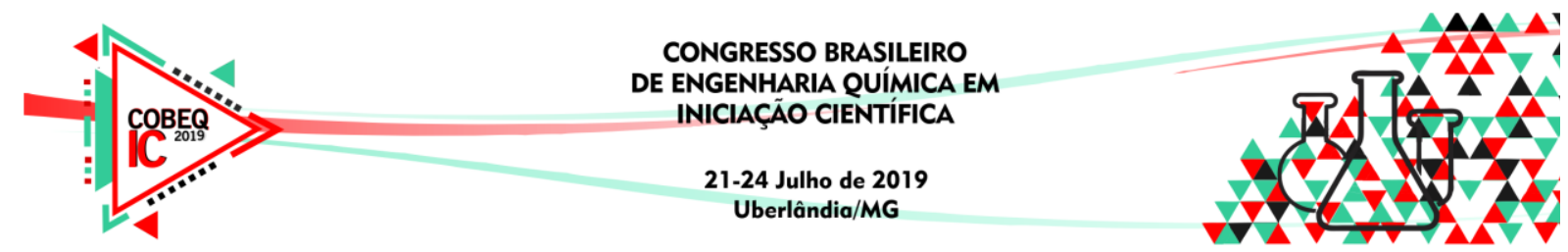

Os dados experimentais obtidos no equilíbrio foram ajustados as isotermas de Langmuir e Freundlich. O modelo da isoterma de adsorção de Freundlich foi a que melhor se ajustou aos dados experimentais $\left(\mathrm{R}^{2}=0,96\right)$ sugerindo a heterogeneidade da superfície do biossorvente e indicando que o processo de adsorção do Azul de Metileno ao biossorvente é favorável $(\mathrm{n}=1,67)$.

\section{CONCLUSÕES}

Os dados obtidos nesse trabalho evidenciaram que a biomassa de A. oryzae é capaz de remover boa parte do corante azul de metileno em solução aquosa, atingindo capacidade de biossorção máxima de $38 \mathrm{mg} \mathrm{g}^{-1}$ em concentrações de $300 \mathrm{mg} \mathrm{L}^{-1}$. Os estudo cinéticos mostraram que o modelo segue a cinética de Pseudo-segunda ordem e a construção das isotermas revelou que a isoterma de Freundlich foi a que melhor se ajustou aos dados experimentais, indicando que a adsorção é favorável $(n>1)$. Estudos adicionais estão sendo realizados a fim de otimizar os parâmetros reacionais, tais como $\mathrm{pH}$, temperatura e dosagem de adsorvente.

\section{AGRADECIMENTOS}

Os autores agradecem o suporte financeiro da CAPES Código Financeiro 001 e FAPEMIG (APQ-01391-18).

\section{REFERÊNCIAS}

OLIVEIRA, S. P.; SILVA, W. L. L.; VIANA, R. R. Avaliação da capacidade de adsorção do corante azul de metileno em soluções aquosas em caulinita natural e intercalada com acetato de potássio. Cerâmica, p.338-344, Cuiabá, jun. 2013.

ANASTOPOULOS, I.; KYZAS G. Z. Composts as biosorbents for decontamination of various pollutants: a review. Water Air Soil Pollut, p. 226-261, 2015.

MAJUNDAR, S. S.; DAS, S. K.; CHAKRAVARTY, R.; SAHA, T.; BANDYOPADHYAY, T. S.; GUHA, A. K. A study on lead adsorption by Mucor rouxii biomass. Desalination, v. 251, p. 96-102, 2010.

SABA, B.; CHRISTY, A. D.; JABEEN, M. Kinetic and enzymatic decolorization of industrial dyes utilizing plant-based biosorbents: a review. Environ Eng Sci, v. 33, p. 601-614, 2016. 